Le « quasi-passé » et la " presque-mémoire » dans Histoire (1967) de Claude Simon

\title{
Wolfgang Asholt
}

\section{(2) OpenEdition}

1 Journals

Édition électronique

URL : http://journals.openedition.org/ccs/671

DOI : $10.4000 /$ ccs.671

ISSN : 2558-782X

Éditeur :

Presses universitaires de Rennes, Association des lecteurs de Claude Simon

\section{Édition imprimée}

Date de publication : 31 décembre 2010

Pagination : 29-47

ISBN : 9782354120771

ISSN : $1774-9425$

\section{Référence électronique}

Wolfgang Asholt, «Le « quasi-passé » et la « presque-mémoire » dans Histoire (1967) de Claude Simon », Cahiers Claude Simon [En ligne], 6 | 2010, mis en ligne le 21 septembre 2017, consulté le 20 avril 2019. URL : http://journals.openedition.org/ccs/671; DOI : 10.4000/ccs.671 


\title{
Le "quasi-passé " et la " presque-mémoire " dans Histoire (1967) de Claude Simon
}

\author{
Wolfgang Asholt, Université d'Osnabrück
}

Dans le chapitre d'introduction de Mimesis, " La cicatrice d'Ulysse ", Erich Auerbach distingue deux types antinomiques de textes : celui d'Homère, décrivant « les événements en les extériorisant » et ceux de la Genèse ou de certains livres de l'Ancien Testament, qui «met[tent] en valeur certains éléments pour en laisser d'autres dans l'ombre ; [d'où] un style abrupt, qui suggère l'inexprimé, l'arrière-plan, la complexité, qui appelle l'interprétation $»^{1}$. Et ce style deviendra, pour lui, un des grands courants de "la représentation de la réalité dans la littérature occidentale ", jusqu'à Proust et Joyce. De son côté, Georg Lukacs, après avoir constaté que « la vie corrige et guide la création des véritables écrivains ", termine son essai, $\mathrm{Le}$ Roman historique sur ces mots :

Le roman historique de notre époque doit donc avant tout nier d'une façon radicale et intraitable son prédécesseur immédiat et extirper énergiquement de sa propre création les traditions de ce dernier. Le rapprochement du roman historique de type classique qui se produit nécessairement dans ces conditions ne sera nullement, comme nos remarques l'ont montré, une simple renaissance de cette forme, une

${ }^{1}$ Erich Auerbach, Mimésis. La représentation de la réalité dans la littérature occidentale, Gallimard, 1994, p. 33. 
simple affirmation de ces traditions classiques, mais, si l'on me permet ici une expression tirée de la terminologie de Hegel, un renouvellement sous la forme d'une négation de la négation. ${ }^{2}$

Il est évidemment difficile de savoir ce que le critique entendait par «négation de la négation », et la négation que représente Histoire de Claude Simon n'est certainement pas celle prévue et espérée par Lukacs. Mais l'assertion de Lukacs a le mérite de souligner que la négation de la négation du " roman historique " peut redevenir, au moins partiellement, un roman historique, et c'est ce que je vais essayer de montrer à travers des commentaires qui sont nécessairement moins ceux d'un simonien que ceux de quelqu'un s'étant penché davantage sur les avant-gardes et le roman contemporain que sur le Nouveau Roman.

\section{Crise du récit littéraire/Crise du récit historique}

Dans l'avant-propos à la deuxième partie de son étude, "Histoire " de Claude Simon, Ecriture et vision du monde, et plus spécifiquement dans la partie consacrée à " Histoire et la contestation de l'histoire ", Véronique Gocel constate : " Le titre Histoire aiguille donc vers la dimension historique du roman et vers l'importante question des rapports de la fiction et de l'histoire. " pour conclure dès le début à "l'impossibilité du roman historique " (Chapitre I, I.) $)^{3}$.

Pour elle, cela tient au " fossé infranchissable » entre "d'un côté l'histoire des manuels d'histoire, qui expose clairement un certain nombre de faits, mais qui ne coïncide pas avec le vécu " et " de l'autre, l'histoire réelle, la réalité des événements vécus, ressentis, mais qui est incohérente parce que l'homme ne maîtrise pas l'instant même qu'il vit. ${ }^{4}$. Joan Brandt, déjà, allait dans le même sens avec un article de 1982, elle aussi concluant d'emblée à cette impossibilité : "For Simon, the conventional relationship between art and

\footnotetext{
${ }^{2}$ Georg Lukacs, Le Roman historique, Payot, 2000, p. 401.

${ }^{3}$ Celia Britton, que Véronique Gocel ne mentionne pas, parle de « the invisibility of History " (chap. 6), dans Claude Simon. Writing the Visible, Cambridge UP, 1987.

${ }^{4}$ Véronique Gocel, Histoire de Claude Simon. Ecriture et vision du monde, Louvain 1996, p. 116-119.
} 
history no longer functions primarily because the notion of history itselfhas become problematical... there is no possibility of an immediate experience of historical events because our perception of those events is never precise or direct but always "confused and chaotic" 5 .

Les deux interprètes - et cela vaut pour nombre d'autres analyses -, ont certainement raison de postuler un antagonisme entre, d'une part, l'Histoire des manuels, d'autre part, la réalité des événements vécus, notre perception de ceux-ci, et surtout leur transposition dans une oeuvre littéraire. Mais la discipline de l'Histoire, avec sa mise en forme des événements passés, est confrontée à des difficultés analogues et a ouvert ce débat dans l'après-guerre. Véronique Gocel évoque à juste titre une "crise du récit " littéraire mais, peutêtre dans une tonalité moins tragique que celle qui nous est propre en tant que littéraires, les historiens ont eu aussi affaire à une crise de leur " récit », et peut-être nos récits en littérature pourraient-ils tirer quelque profit à intégrer le débat historique dans ses réflexions, surtout lorsque celles-ci portent sur le " roman historique ». Joan Brandt admet pourtant la persistance d'une certaine mimesis quand elle écrit que Simon :

does not radically change the mimetic principle upon which the traditional novel is based, for Simon continues to postulate the possibility of immediate representation, although in this case it is not the outside world that the writer refers but to that which is 'en lui à l'instant où il décrit'. ${ }^{6}$

Autrement dit, Claude Simon admettrait quelque chose comme une « écriture immédiate » ne reflétant plus la réalité (devenue histo-

5 Joan Brandt, "History and art in Simon's Histoire », in Romanic Review 73 (1982), p. 373 ; trad. : "Pour Simon, la relation conventionnelle entre l'art et l'histoire ne peut plus fonctionner, tout simplement parce que la notion d'Histoire elle-même est devenue problématique, il n' y a aucune possibilité d'expérience immédiate des événements de l'Histoire parce que notre perception de ces événements n'est jamais précise ou directe, mais toujours confuse et chaotique. » ${ }^{6}$ Joan Brandt, ibid., p. 374 ; trad. : « Simon ne change pas radicalement le principe mimétique sur lequel le roman traditionnel est fondé car il continue à postuler la possibilité d'une représentation immédiate, bien que, dans ce cas, ce ne soit pas au monde extérieur que le romancier se réfère mais à « ce qui est en lui à l'instant où il le décrit »; la citation en français est tirée de l'interview avec Bettina Knapp. 
rique), mais simplement la mémoire produisant de tels instantanés. Si c'est la mémoire qui fait resurgir ces instantanés, c'est pourtant l'écriture qui les met en forme et les organise. La simultanéité déjà limitée de la mémoire est transformée en linéarité romanesque, ce qui mène Joan Brandt à la thèse suivante : "One can only conclude, then, that art, in contrast with the theories of Simon, functions in the same manner as history. [...]. The narrator and the historian can never find the proper perspective; they are either too close to the event or too far away. $\gg^{7} L^{\prime} œ u v r e$ de Claude Simon présente l'avantage de montrer cette impossibilité, le sujet-narrateur lui-même en fait l'expérience. Mais comme c'est la seule possibilité dont l'Art et l'Histoire disposent pour rendre compte de ce qui se passe en dehors d'eux (et il y a un dehors de la littérarité dans Histoire), l'effet de réel est conditionné et produit par cette perspective. Brandt peut donc conclure : " they both [1'Art et l'Histoire] create a fiction that replaces the real by imposing an artificial and linear order $\rangle^{8}$.

Mais cette présence de l'Histoire dans Histoire - ne serait-ce que par son absence même - est souvent contestée par la critique simonienne. Dans le dossier consacré au roman par la Revue des Lettres Modernes en 2000, Ralph Sarkonak revendique pour ce roman une littérarité auto référentielle : "[l] ibéré de la représentation, le langage $\mathrm{du}$ texte peut revendiquer sa matérialité et de nouveau s'adonner aux jeux enfantins qui sont à la base de la créativité. $"{ }^{9}$ Cette apologie du travail sur le signifiant ne tient pas compte du résultat de ce jeu, car pour Claude Simon « les mots possèdent [...] ce prodigieux pouvoir de rapprocher et de confronter ce qui, sans eux, resterait épars. »C'est grâce à ce pouvoir que devient possible « l'aventure singulière du narrateur », qui découvre «à tâtons le monde dans et par

${ }^{7}$ Ibid., p. 383 ; trad. : " on peut simplement conclure que cet art, en opposition avec les théories de Claude Simon, fonctionne de la même façon que l'histoire. Le romancier, comme l'historien, ne peuvent trouver la perspective adéquate, ils sont toujours trop près ou trop loin de l'événement $»$.

${ }^{8}$ Ibid., p.384 ; trad.: « l'art et l'hitoire, tous deux, créent une fiction qui remplace le réel en imposant un ordre artificiel et linéaire».

${ }^{9}$ Ralph Sarkonak, "Le texte polyphonique ", dans Claude Simon 3 - lectures de Histoire, "La revue des Lettres modernes ", Minard 2000, p.7 ; cette idée avait d'ailleurs été formulée plus tôt par Antony Pugh dans la première monographie consacrée à l'œuvre. 
l'écriture ", ainsi que le postule la conclusion de la Préface à Orion aveugle ${ }^{10}$. Ce principe de " rapprochement» et de " confrontation» invoqué par Simon, correspond presque à la définition de l'image par Pierre Reverdy ( Plus les rapports des deux réalités rapprochées seront lointains et justes, plus l'image sera forte, plus elle aura de puissance émotive et de réalité poétique. $\|^{11}$ ) ; quant à l'hypothèse de la " découverte du monde ", elle pourrait indiquer que la " libération de la représentation "n'est pas une fin en soi, trouvant son accomplissement exclusivement dans la matérialité du texte, mais que ce texte participe aussi de ce qu'on a appelé dans un débat allemand dernièrement un Lebenswissen, un "savoir de la vie» ${ }^{12}$. Roland Barthes, dans sa "Leçon " inaugurale au Collège de France (janvier 1977), a souligné l'importance de ce savoir pour la littérature et pour la critique littéraire en localisant « les forces de liberté qui sont dans la littérature " dans le " travail de déplacement " que l'écrivain exerce sur la langue. Grâce à ce déplacement/rapprochement, selon Barthes, " la littérature fait tourner les savoirs » et dans la littérature, « le savoir réfléchit sans cesse sur le savoir, selon un discours qui n'est plus épistémologique, mais dramatique. $»^{13}$

\section{Le quasi-passé de la fiction et ses potentialités}

Or l'Histoire fait partie de ce savoir de la littérature. ${ }^{14}$ Depuis Metahistory (1973) de Hayden White, la plupart des historiens tombent d'accord pour définir leur discours comme résultat d'un « effet de narration ", et pourrait-on ajouter, en dernière instance comme celui d'un travail de la fiction. Avec mon collègue allemand Andreas

${ }^{10}$ Claude Simon, "Préface à Orion aveugle (1970) », dans Euvres, Gallimard, "Bibliothèque de la Pléiade» 2006, p. 1182-1183.

${ }^{11}$ Pierre Reverdy, "L'Image », dans Nord-Sud. Self-Defence et autres, Ecrits sur l'art et la poésie, Flammarion, 1975, p. 73.

${ }^{12}$ CfOttmar Ette, "Literaturwissenschaft als Lebenswissenschaft ", dans Lendemains 125 (2007), p. 3-35, et le prolongement de ce débat dans les numéros 126 à 128 (2007) puis 129 (2008) de la revue.

${ }^{13}$ Roland Barthes, Leçon, dans Euvres complètes, vol. 3, Seuil, 1994, p. 804 -805. ${ }^{14}$ Je renvoie dans ce contexte au volume 10 des Ecritures contemporaines, édité par Dominique Viart et consacré aux Nouvelles écritures littéraires de l'Histoire, La Revue des Lettres modernes, Minard 2009. 
Kablitz, on peut supposer que « les qualités structurelles de la narration sont liées aux qualités structurelles de l'histoire même $»^{15}$. Mais ces qualités inhérentes à la narration ne concernent pas seulement l'Histoire en tant que discipline caractérisée par la narrativité de son discours, elles concernent aussi la littérature. Kablitz voit « l'élasticité de la narration comme médium de la production d'une variété infinie de relations " (on pourrait aussi bien parler de déplacements que de rapprochements) ; la fiction narrative devient alors un instrument privilégié pour dégager " la complexité de l'Histoire même et du réseau relationnel qui la constitue. ${ }^{16}$ Pour Kablitz, comme, depuis les années 1980, pour les théoriciens de la memoria en Allemagne, avant tout Aleida et Jan Assmann ${ }^{17}$, la littérature occupe une place privilégiée dans la mémoire culturelle : elle met en scène la mémoire d'une époque concrète, s'offre comme lieu de formation de cette mémoire, voire de concurrence entre plusieurs types de mémoire, mais c'est aussi une instance permettant l'observation critique des processus et des procédés de la mémoire culturelle.

Paul Ricœur, lui aussi, s'est interrogé, dans le volume consacré au «Temps raconté » de Temps et récit (1983-1985) sur « l'entrecroisement de l'histoire et de la fiction », en distinguant trois types de mimésis (signes, règles et normes de l'action symboliquement préfigurés, transmises par les structures narratives et portés par l'expérience esthétique des lecteurs). Comme Kablitz, Ricœur développe sa conception du récit, en discutant les thèses de White. Mais pour lui, il s'agit d'un côté, " [de ne pas] effacer la frontière entre la fiction et l'histoire ", et de l'autre, de combattre le "préjugé " " selon lequel la littérature d'imagination, parce qu'elle use constamment de

${ }^{15}$ Andreas Kablitz, " Geschichte - Tradition - Erinnerung ? Wider die Subjektivierung der Geschichte », dans Geschichte und Gesellschaft 32 (2006), p. 225 (c'est moi qui traduit, W.A.).

${ }^{16}$ A. Kablitz, op.cit., p. 237.

${ }^{17}$ Par exemple, Aleida Assmann, Construction de la mémoire culturelle, Éditions de la Maison des Sciences de l'Homme, 1994 ; Erinnerungsràume. Formen und Wandlungen des kulturellen Gedächtnisses, München, Beck 1999, et Jan Assmann, dernièrement, La mémoire culturelle : Ecriture, souvenir et imaginaire politique dans les civilisations antiques, Aubier, 2010. 
la fiction, doit être sans prise sur la réalité. $\rangle^{18}$ Et Ricœur franchit, me semble-t-il, un pas de plus que Kablitz quand il affirme que :

l'intentionnalité historique ne s'effectue qu'en incorporant à sa visée les ressources de fictionnalisation relevant de l'imaginaire narratif, tandis que l'intentionnalité du récit de fiction ne produit ses effets de détection et de transformation de l'agir et du pâtir qu'en assumant symétriquement les ressources d'historicisation que lui offrent les tentatives de reconstruction du passé effectif. ${ }^{19}$

Pourtant, malgré les « échanges intimes entre historicisation du récit de fiction et fictionnalisation du récit historique ", Ricœur admet une place et un statut spécifique de la fiction littéraire : "Le quasi-passé de la fiction devient ainsi le détecteur des possibles enfouis dans le passé effectif $\gg .{ }^{20}$ La question qui se pose à partir de cette relation entre Histoire et littérature est celle du statut de la narration et de la fiction dans l'un comme dans l'autre genre. Même si l'on peut mettre en question la validité des « tentatives de reconstruction du passé effectif" pour une grande partie de la littérature après le Nouveau Roman, le constat que "[l]e quasi-passé de la fiction devient ainsi le détecteur des possibles enfouis dans le passé effectif " me semble difficilement contestable. Ce sont ce " quasi-passé » et ces « quasi-possibles » qui font passer un roman comme Histoire « d'un prétendu savoir historique à un travail de mémoire ; de la reconstitution monumentale au procès de l'anamnèse. $»^{21}$

\section{Mémoire de qui ? Ou de quoi ?}

Mais si le travail de mémoire se trouve être au centre de l'œuvre de Simon, ce que confirment, surtout depuis les années 1990, les titres

\footnotetext{
${ }^{18}$ Paul Ricœur, Temps et récit, vol. 3, Le temps raconté, Seuil-Points, 1985, p. 279280.

${ }^{19}$ Ibid., p. 185.

${ }^{20}$ Ibid., p. 347. Le " quasi-passé » de Ricœur renvoie au " quasi-jugement des constatations dans les œuvres littéraires » (" quasi-urteilsmäBige Behauptungssätze in literarischen Werken ») dont parle Roman Ingarden dans Das literarische Kunstwerk, Tubingen, Niemeyer, 1960 (première édition 1931), p. 182.

${ }_{21}$ Mireille Calle-Gruber, Le Grand Temps. Essai sur l'ouvre de Claude Simon, Presses du Septentrion 2004, p. 27-28.
} 
de nombre d'études (Une mémoire inquiète. La Route des Flandres de Claude Simon de Dominique Viart (1997) ou Chemins de la mémoire de Mireille-Calle-Gruber (1993) ou plus souvent encore de chapitres (comme "Matières de mémoire " chez Didier Alexandre, Le magma et l'horizon (1997)), quelle est la fonction de ce travail de mémoire ? Et de quel type de mémoire s'agit-il?

Est-ce que c'est exclusivement "une précaire et monumentale reconstruction d'un moi vivant à partir de ses ruines ", constat avec lequel Jean Starobinski termine sa magistrale analyse de « La Journée dans Histoire $\|^{22}$ ? Donc d'un "quasi-passé » tout à fait personnel opposé à un ou au "passé effectif» et d'une presque-mémoire du même type ? Starobinski, qui répète les termes du champ sémantique de « mémoire-memoria » (par exemple : « rituels remémorés », « la mémoire ", " le rite remémoré ", " image remémorée », "Telle est notre mémoire $»^{23}$ ) parle cependant, à la dernière page de l' essai, de «l'un des horizons de ce passé resurgi qu'est le naufrage du temps », ce qui l'amène au constat que «le travail inépuisable qui se développe dans Histoire pourrait avoir pour nom « deuil et mélancolie ${ }^{24}$. Ce « deuil " et cette " mélancolie " pourraient être ceux du sujet (autofictionnel ou non) de Histoire.

Toutefois, l'infinité de faits historiques mentionnés ou intégrés comme collages et fragments dans le texte semble indiquer une tentative de dépassement d'une expérience uniquement personnelle, même si Claude Simon assure, peu après la publication d'Histoire, que " ce texte ne correspond qu'à une seule chose, ce qui se passe dans l'écrivain au moment où il écrit. ${ }^{25}$ L'écrivain n'en représente pas moins une " histoire vécue», personnelle, familiale mais aussi historique et sociale, ce dont témoignent de nombreux passages du roman. Leur caractère répétitif, parfois obsessionnel, confirme la " pré-existence » de certaines " choses " par rapport à l'écriture, " choses " qui constituent les références de la "presque-mémoire " de l'écrivain. Cette approche se trouve aussi justifiée par ce que Per

\footnotetext{
${ }^{22}$ Jean Starobinski, "La Journée dans Histoire », dans Sur Claude Simon, Minuit, 1987 , p. 32.

${ }^{23}$ Ibid., p.16, p. 17, p.18, p.21, p. 29.

${ }^{24}$ Ibid., p. 32.

${ }^{25}$ Bettina Knapp, «Document. Interview avec Claude Simon », dans Kentucky Romance Quaterly 16(1969), p. 182.
} 
Nykrog ${ }^{26}$ désigne malicieusement par la formule «Croquis pour une lecture naïve " d'Histoire. Selon lui, nous assistons dans ce roman à une " auto-analyse " où à la fin, le sujet-narrateur " a réussi à faire face à ses traumatismes " et " peut enfin trouver le sommeil », une interprétation compatible avec la « reconstruction d'un moi vivant à partir de ses ruines " pour reprendre Starobinski. Mais le lecteur qui suit cette entreprise, ne se borne pas à « reconstituer [...] la série d'événements épars qui [...] constituent pour lui, à ce moment-là, l'essentiel de son histoire», il constate que «ce roman peut aussi être lu comme une réflexion sur l'Histoire, avec majuscule. $»^{27} \mathrm{Si}$ le roman a pour arrière-plan l'histoire des deux premiers tiers du $\mathrm{XX}^{\circ}$ siècle, il en privilégie quelques moments spécifiques : le monde de la Belle Epoque, surtout à travers les cartes postales, les années 1930 jusqu'à la défaite de 1940, et le temps de la narration qui se situe vers le milieu des années 1960. C'est une histoire aussi bien vécue que traversée et supportée mais on peut dire avec Nykrog, « que ce n'est que durant cette journée de retour sur lui-même que [le narrateur] la rencontre vraiment. ${ }^{28}$ Etant donné que le protagoniste a presque exclusivement subi cette histoire de son siècle et que la rencontre possible avec l'événement en train de se faire a profondément échoué dans le Barcelone de l'année 1936 - ce qui explique peut-être aussi la polémique contre l'auteur de L'Espoir, dans le Discours de Stockholm, ou contre Orwell, dans une interview ${ }^{29}$, - ce ne peut être qu'un résidu informe, incompréhensible et absurde (voir ses " explications » de l'aventure espagnole à l'oncle Charles ou à son ancien camarade de classe et ami Lambert), le fameux " magma informe " dont il est parlé dans Orion aveugle... Ce n'est donc que rétrospectivement qu'il peut faire face à ses traumatismes personnels et historiques - et dans

${ }^{26}$ Per Nykrog, "Veuf. Croquis pour une lecture naïve », dans R. Sarkonak, La Revue des Lettres Modernes, Minard, 2000.

${ }^{27}$ Ibid., p. 22 et 34.

${ }^{28}$ Ibid., p. 35.

${ }^{29}$ Claude Simon y parle « de terrifiants récits d'aventures à conclusions optimistes ou désespérées, et aux titres de vérités révélées comme par exemple La condition humaine, L'espoir ou Les chemins de la liberté... " (Discours de Stockholm, Minuit 1987, p. 15). Et dans une interview de 1985, il déclare : "ce qu'Orwell a mis six mois à comprendre, je l'ai vu en quinze jours : c'était voué à l'échec. ", Interview avec Marianne Alphant, Libération, 10 déc. 1985. 
Histoire, il est souvent difficile de séparer le personnel de l'historique - en les appréciant et les valorisant comme "deuil et mélancolie» vis-à-vis de la crise et de la rupture de civilisation que représente l'histoire du XX' siècle.

Dans une étude magistrale des " espaces de mémoire " (Gedächtnisräume), que les Cahiers Claude Simon ont heureusement traduite, Rainer Warning analyse le fonctionnement et la fonction de ces espaces qui «n'aident plus le Moi à récupérer rapidement des images bien ordonnées, mais s'ouvrent à des séquences d'images discontinues et, par là, à une profondeur temporelle dans laquelle le Moi se remémorant se perd pour toujours $»^{30}$, il en résulte chez Simon " une rhétorique déconstructive qui voit, dans l'écriture de mémoire du Moi, l'histoire de sa mort $\|^{31}$. Dans ces « espaces de mémoire » ou clans cette « presque-mémoire se situe le quasi-passé du « magma informe $\|^{32}$. Pour Warning, les guerres du $\mathrm{XX}^{\circ}$ siècle chez Simon représentent "une intertextualité incroyablement riche $\|^{33}$, mais les références extralittéraires, s'il y en avait, ne l'intéressent pas. Avec leur « magma informe», les romans de Simon sont pourtant « riches en traces du vécu et en allusions aux événements majeurs de notre commune histoire européenne », comme le constate Anthony Cheal Pugh dans un article au titre significatifde "Simon et la route de la référence $\|^{34}$. Une analyse qui se situerait sur cette " route de la référence " et essaierait une " lecture " des traces pourrait donc montrer qu'il s'agit non seulement d'un travail de deuil personnel où tout serait l'histoire de la mort du moi, mais aussi d'un travail qui hérite, comme l'exprime Starobinski « de toute l'histoire du monde » ${ }^{35}$. L'élasticité de la narration romanesque et sa production d'une variété

${ }^{30}$ Rainer Warning, "Les espaces de mémoire de Claude Simon, La Route des Flandres » (paru en allemand en 1991), trad. dans Cahiers Claude Simon I, 2005, p. 115 .

${ }^{31}$ Ibid., p. 122-123.

${ }^{32}$ Il serait intéressant de voir comment Rainer Warning interprète la «précaire et monumentale reconstruction d'un moi vivant à partir de ses ruines " de Starobinski, mais Warning ne mentionne pas l'étude de Starobinski parue en 1987.

${ }^{33}$ Rainer Warning, ibid., p. 126.

${ }^{34}$ Anthony Cheal Pugh, "Simon et la route de la référence " dans Revue des Sciences humaines ${ }^{\circ} 220$, Guy Neumann dir., 1990, p. 27.

${ }^{35}$ J. Starobinski, op. cit., p. 32. 
infinie de relations, serait donc un instrument privilégié pour dégager dans ce quasi-passé romanesque « la complexité de l'Histoire même et du réseau relationnel qui la constitue.» Le roman deviendrait alors "le détecteur des possibles enfouis dans le passé effectif» dont parle Ricœur et la reconstruction du moi (d'un moi chargé de toute l'histoire du monde) est en même temps une reconstruction de ces possibles démentis par l'histoire, un travail qui porterait à juste titre le nom de " deuil et mélancolie».

Dans la quatrième de couverture des Géorgiques \{1981), Claude Simon explique : " trois personnages vivent des événements et des expériences qui semblent se répéter, se superposer ». Dans Histoire, ce sont plutôt trois périodes privilégiées qui remontent à la mémoire du protagoniste et déclenchent le travail de deuil : celle de la Belle Epoque et de la Grande Guerre, celle des années 1930 et de la défaite de 1940, et celle de l'époque de la narration, donc du plein essor des "Trente glorieuses », et il arrive souvent que ces périodes soient reliées par la co-présence de figures comme celles de l'oncle Charles, du cousin Paulou ou de l'ami de classe Lambert. Toutefois, le statut narratif de ces périodes diffère: pour la Belle Epoque, les « cartes postales » figurent un collage, grâce à l'intégration textuelle de documents - réels ? -, mais aussi la métaphore des plissements tectoniques d'une mémoire qui ne peut ni ne veut reconstruire la linéarité chronologique. Et pour l'époque contemporaine, il s'agit plutôt d'un effet de contiguïté entre les passages consacrés à Paulou et à Lambert vers la fin du roman dont résulte une métonymie des « vainqueurs" de l'histoire, étonnamment actuelle.

\section{La « cicatrice d'Ulysse " mémorielle}

Mais il me semble que les passages concernant le Barcelone des premières semaines de la Guerre d'Espagne, représentent la référence personnelle et collective centrale pour le travail de deuil de la " presque-mémoire " qu'entreprend Histoire, Barcelone devenant ainsi une " cicatrice d'Ulysse » mémorielle comme la « route des 
Flandres » le sera dans d'autres romans ${ }^{36}$. En général, l'auteur est assez discret sur son engagement pendant ce "moment crucial de l'histoire de l'Europe du $\mathrm{XX}^{\mathrm{e}}$ siècle », comme Jorge Semprun qualifie cette guerre ${ }^{37}$. Et pourtant, le Barcelone de la révolution et de la guerre civile occupe une place importante dans l'œuvre de Simon : Le Palace (1962) y est entièrement consacré ainsi que le quatrième chapitre des Géorgiques ou nombre de passages du Jardin des Plantes; ces " chers souvenirs sont plus lourds que les rocs. ${ }^{38}$ Dans l'interview avec Mireille Calle-Gruber, Simon décrit son expérience à Barcelone par la litote du " curieux » ou du "voyeur»; il est un peu plus explicite, dans la notice de l'année 1988, sur les conditions qui l'ont amené à "faire passer clandestinement en Espagne une cargaison d'armes » et à « entrevoi[r] alors le monde des trafiquants et des horsla-loi »; il relativise la signification de l'épisode en le commentant comme une "intéressante expérience » que "son dilettantisme, son manque de véritables convictions, et partant d'efficacité, ne lui font pas renouveller ». Une phrase, renforçant les litotes mentionnées, pourrait cependant donner un indice de la signification personnelle de cette " participation 》 relativisée : "Il se perçoit confusément comme un imposteur et rentre en France après deux semaines..${ }^{39}$

${ }^{36}$ Jacques Leenhardt a consacré au Barcelone du Palace une intervention remarquable lors de la semaine "Claude Simon » de Cerisy en 1974, il a cherché à montrer "comment la problématique de l'écriture s'élabore elle-même à travers les textes comme partie intégrante des idéologies. " (« Discussion », dans Jean Ricardou éd., Claude Simon, 10/18, 1975, p. 147. On a vu quelle critique une telle position avait provoqué de la part de Jean Ricardou ( L'effet de vraisemblance, dans un texte, a un effet second : celui de faire disparaître le texte comme texte par l'abolition de sa matérialité dans la transparence du sens. », ibid., p. 141). C'est évidemment à une objection de ce type que s'expose mon article, un regard critique tel que celui-ci excluant la présence textuelle de tout « quasi-passé » et toute " presque-mémoire ».

${ }^{37}$ Discours prononcé lors de l'ouverture de l'exposition « Espana en el corazón », Berlin (Staatsbibliothek), 6 février 2006. Voir W. Asholt, R. Reinecke, S. Schlünder (éd.), Der Spanische Biirgerkrieg in der DDR. Strategien intermedialer Erinnerungsbildung, Francfort, Vervuert, 2009, p. 27- 32.

${ }^{38}$ Charles Baudelaire, "Le Cygne ", dans Les Fleurs du Mal, Euvres, vol. 1, Gallimard, "Bibliothèque de la Pléiade", 1975, p. 86.

${ }^{39}$ Claude Simon, " Notice rédigée en 1988 ", dans CEuvres, Gallimard, "Bibliothèque de la Pléiade», p. LXX. 
L'épisode de Barcelone dans Histoire occupe aussi une place centrale dans la succession des douze chapitres du roman, les chapitres cinq à sept lui étant consacrés, en alternance avec d'autres motifs ${ }^{40}$. Deux procédés différents servent à la visualisation de la ville : d'une part l'évocation d'une gravure à l'aquatinte représentant une vue panoramique, et de l'autre la description détaillée de l'épisode d'un combat de rue, déclenché pourrait-on croire par une panne de voiture. La "VUE GENERALE DE BARCELONE » représente " la ville jaunâtre étalée entre une mer pâle et sa ceinture de collines, semblable à ces panoramas que l'on peut voir sur les couvercles des boîtes de dattes [..] " (Hist. p. 160) et le sujet nous donne moins une description qu'une interprétation de la forme de cette ville. C'est un panorama, "se réduisant à la fin à de simples signes » et ce panorama se transforme en matériel même de l'écriture : « il s'écoulait un temps assez long avant que les yeux [...] perçoivent de faibles bruits [...] comme s'il leur avait fallu un délai considérable pour traverser ce magma informe" (Hist., p. 163) Comme l'écrit Karlheinz Stierle, dans La capitale des signes, "Ce n'est qu'à partir du moment où le langage du texte répond aux langages de la ville que ceux-ci peuvent être pénétrés et questionnés $»^{41}$ et c'est dans le texte consacré à sa "vue générale » que la ville peut se voir elle-même ; au « magma informe " de la description correspond donc celui de la structure narrative. Le sujet comprend « que l'activité visible se déroulait pour ainsi dire dans une zone inférieure, sous-jacente au bruit émis non par des humains [...] mais par la cité elle-même. " (Hist., p. 163) Pour David Carroll, cette représentation visuelle " undermines the rigid synchronic/diachronic opposition by challenging the way in which space is enclosed and time ordered [...] Time is always-al-

${ }^{40}$ L'article de Pascal Mougin « Histoire. L'aventure espagnole comme quête oedipienne " tient les promesses du titre en excluant toute lecture " historique " comme « niveau superficiel de lecture " et insistant sur « la connexion du tabou majeur (le coït parental) à l'échec de l'expérience guerrière " (dans Lectures de "Histoire ", Revue des lettres modernes, Claude Simon 3, Paris-Caen 2000, p. 126). ${ }^{41}$ Karlheinz Stierle, La capitale des signes. Paris et son discours, Maison des sciences de l'homme, 2001, p. 35. 
ready at work within the space which the eye looks and listens to. $\gg^{42}$ Ce que nous dit le bruit sous-jacent, monté des profondeurs de la ville, un bruit et un frémissement que nous connaissons depuis le début du roman, et que les yeux écoutent, c'est que c'est « une ville vouée, consacrée à l'avance [...] à la violence, aussi irrémédiablement qu'un certain dosage de salpêtre et de charbon mélangé et comprimé dans un tube d'acier est destiné à exploser» (Hist., p. 161) ${ }^{43}$. Avec cette allusion aux bombes fabriquées par les anarchistes, le deuxième motif-clé lié à Barcelone est déjà évoqué, celui qui correspond à la consécration séculaire de cette ville, celui du " magma informe»devenu éruptif et explosif.

Avec l'épisode consacré au combat de rue banal, dérisoire et incompréhensible, pour les participants, pour les observateurs et pour les lecteurs, la question de la guerre civile est abordée, une guerre qui, à Barcelone à ses débuts en été 1936, et de nouveau en 1937, est extrêmement compliquée avec les combats entre les défenseurs de la République et les troupes putschistes d'abord mais surtout, un peu plus tard, avec les combats entre anarchistes et trotskistes d'un côté (mais, aussi, parfois entre eux) et les communistes de l'autre, auquel s'ajoute la hantise de la "cinquième colonne " qu'évoque aussi Le Palace. Le sujet introduit ces " chers souvenirs " d'une ville en guerre par la discussion rapportée avec son oncle Charles, qui est emblématique non seulement de l'interprétation des événements, mais de la conception de l'Histoire tout court

Mais raconte-moi encore ça. Si tu n'es pas encore arrivé à décider ce que tu allais faire là-bas essaie au moins de te rappeler non pas comment les choses se sont passées (cela tu ne le sauras jamais - du moins celles que tu as vues : pour les autres tu pourras toujours lire plus tard

42 David Carroll, The Subject in Question. The languages of theory and the strategies offiction, Chicago UP, 1982, p. 157 et p. 159; trad.: «défait l'opposition rigide entre synchronie et diachronie en remettant en cause la manière dont l'espace est inclus dans le texte et le temps ordonné [...]. LeTemps [...] est toujours déjà au travail dans l'espace que l'oeil regarde et écoute."

${ }^{43}$ Cette image est, dans la suite du récit, liée à celle de la modernité même : le sujet évoque " un acide corrodant, jaunâtre et sulfurique s'échappant en même temps que les fumées de ses usines qui retombaient, s'affalaient sur elle [la ville] impuissantes à percer le ciel de plomb où s'étiraient quelques écharpes de brume elles-mêmes rousses captives. " \{Hist., p. 161) 
les livres d'Histoire) mais comment... [suit une parenthèse de presque une page consacrée à ce qui se passe pendant la discussion avec l'oncle] ...et toi collé aplati contre ou plutôt incrusté dans ce mur ... (Hist., p. 172-173)

Le « raconte-moi encore » rappelle le « mais exactement ? " trois fois répété dans la troisième partie (Hist., p. 78, p. 87 et p. 93). Nous non plus ne saurons donc pas " comment les choses se sont (plus ou moins exactement) passées " et nous sommes renvoyés aux livres d'Histoire pour ce qui n'a pas été vu. Mais nous en apprenons encore moins sur l'alternative indiquée par le deuxième « mais comment. .. » suivi des trois points, à la manière de Céline, qui ne trouve pas de suite syntaxique et fonctionne comme une ellipse sans issue, la "presque-mémoire " se transformant en léthé. Au lieu de cette alternative indiquée et promise par le discours de l'oncle mais démentie par celui-ci même, nous sommes plongés dans le commentaire de la situation du sujet fait par cet oncle : " tu n'étais plus un homme capable je ne dis pas de comprendre mais tout au moins de voir ce qui se passait : seulement quelque chose de fluide, translucide et sans consistance réelle, comme nous sommes d'ailleurs tous plus ou moins dans le présent. » (Hist.p. 173) Oncle qui en devient presque un récitant - raisonneur... Et ce n'est qu'après cette appréciation générale des limites de tout savoir historique, lorsqu'il prétend interpréter la signification des événements vécus, que le sujet peut aborder le « quasi-passé », non sans prévenir le lecteur : "recommençons premièrement deuxièmement troisièmement impossible " (Hist., p. 175). Ce qui suit est le " magma informe» d'un échange de tirs dans une avenue bordée d'arbres autour d'une voiture arrêtée, voiture réquisitionnée par des hommes armés de l'U.H.P., c'est-à-dire une des fractions révolutionnaires espagnoles ${ }^{44}$. Après le bruit des fusils et celui des cris se font entendre des éclats de voix, correspondant à une discussion entre deux groupes opposés, puis l'on s'installe de nouveau dans les cafés et la voiture repart. Si on essayait de dessiner ce qui se passait à ce carrefour, commente l'oncle, ce serait : « un en-

${ }^{44}$ U.H.P. (Union de Hermanos Proletarios) fut un essai de Front populaire en Asturies, lors des grèves d'octobre 1934. Mais en dépit de quelques tentatives esquissées, une alliance semblable n'a jamais pu être réalisée à Barcelone, l'inscription sur la voiture est donc un commentaire ironique des dissensions révolutionnaires. 
trecroisement, un enchevêtrement de lignes pointillées (je veux dire chaque fois que tu entendais ce bruit, ce tac-tac de machine à écrire) qui partaient d'un peu partout vers un peu partout " (Hist., p. 180) Ces signes géométriques deviennent des signes textuels dont résulte la signification de l'épisode. Ce qu'écrit ou dessine cette machine à écrire, c'est « l'Histoire en train de se faire » comme l'oncle le dit explicitement.

\section{Vers un Jetzzeit}

Le sujet, aux traits autobiographiques manifestes, subit donc, pendant son bref séjour à Barcelone, la rencontre avec l'Histoire en train de se faire. A la différence de la plupart des volontaires étrangers qui combattent du côté de la République, il ne sait pas trop bien ce qu'il allait faire là-bas, "[a]vec ces bandits et ces assassins... " \{Hist., p. 188), comme les qualifie sa cousine Corinne. Sans l'idéal d'une conviction idéologique, à la différence de l'Italien, l'hommefusil, ou du maître d'école du Palace, une idéologie qui permet l'illusion d'une vue globale et explicative des événements, le sujet ne peut donc interpréter ce qu'il vit que comme un itinéraire, non seulement dénué de sens mais absurde et violent à la fois. De cette première confrontation avec l'Histoire, - étant donné $\Gamma$ âge du protagoniste -, résulte la cicatrice d'un scepticisme pessimiste vis-à-vis de tout progrès historique. Avant qu'il ait pu le développer, le sujet " a perdu ce qui ne se retrouve / Jamais, jamais!" (les espoirs liés par d'autres à la révolution espagnole), et il réagit dorénavant avec mélancolie et par un travail de deuil.

Dans la biographie du sujet, cette expérience précède celle de l'époque du temps de la narration, c'est-à-dire des années 1960. L'épisode des combats de Barcelone du sixième chapitre d'Histoire est d'ailleurs intégré à une scène de restaurant des années 1960 : on y perçoit d'abord la caricature d'une bourgeoisie aisée et bête et, à l'occasion d'une description d'illustrations de billets sortis pour une addition, un démenti complet des espoirs de la révolution espagnole. Mais l'épisode de Barcelone précède aussi l'apparition de l'autre support mémoriel que sont les cartes postales de la Belle Epoque, retrouvées grâce à la vente d'une commode, donc au moment du temps de la narration. Et surtout, elle représente une "presque-mé- 
moire » très personnelle, liée à l'un des événements majeurs de l'histoire du $\mathrm{XX}^{\mathrm{e}}$ siècle.

La Guerre d'Espagne, rappelons-le, est devenue rapidement un lieu d'engagement et de mémoire, surtout à cause du soutien actif de nombreux intellectuels étrangers du côté de la République espagnole, et dès le début de cette guerre se développent une littérature mais aussi un art engagés. Même un Beckett a répondu à une enquête anglaise en 1937 avec un seul mot écrit en majuscules et avec les deux points d'exclamation à l'espagnole: «! UPTHEREPUBLIC ! $\rangle^{45}$. Cette littérature, quand elle n'est pas tout simplement une apologie de l'héroïsme républicain, se veut au moins un lieu de mémoire de l'espoir que représentait la résistance contre le fascisme européen, souvent dans le sens de ce que Lukacs a désigné par « roman historique de type classique ». Face à cette littérature de témoignage, les passages consacrés par Claude Simon au Barcelone de 1936 ne laissent pas de place pour le plus mince discours engagé. L'expérience de "l'Histoire en train de se faire » est accompagnée et approfondie dans le roman d'un travail de mémoire qui, quand la guerre est finie "trente ans après », ne permet plus la moindre illusion sur la signification de cette Histoire. C'est ce travail de mémoire qui produit la mélancolie et le deuil dont parle Starobinski. Mais sa référence est l'expérience d'une situation vécue par le protagoniste et, comme le dit Starobinski : "Une référentialité de cette sorte, dans les limites mêmes de l'œuvre de fiction, ne contrevient pas à la primauté du texte. " Et Claude Simon confirme cette "référentialité » lorsque Lucien Dällenbach lui (pro-)pose la question-suggestion suivante : «Dans Histoire l'écriture de l'histoire, du roman qui se fait sous nos yeux, n'est-elle pas plus importante que l'histoire ? », il répond : « Je répète [ !] : il y a toujours ambiguïté. Cela fonctionne toujours plus ou moins à deux niveaux. $»^{46}$

Cette référentialité est celle d'un "présent complexe, multiple

${ }^{45}$ Samuel Beckett, dans Authors take sides on the Spanish War, London, Left Review, 1937.

46 Lucien Dallenbach, «Attentes et stimuli » (Interview de février/mars 1987), dans Claude Simon, Seuil 1988, p. 174. Et dans une interview avec Jean-Claude Lebrun, Simon explique ainsi sa méthode « ambiguë » : «Peut-être que ça permet aussi de dégager davantage de sens ? Si nous abordons la question du sens !...» («L'Atelier de l'artiste», dans Révolution, 29 septembre1989, p. 37). 
celui que Benjamin nomme Jetztzeit $\aleph^{47}$ note Peter Janssens qui renvoie aux trois temps (du présent) selon Saint-Augustin que mentionne, dans son essai, Mireille Calle-Gruber : « le présent du passé, le présent du présent, le présent du futur. $»^{48}$ Et Claude Simon définit cette relation ainsi : «Le souvenir est à la fois antérieur à l'écriture et suscité (ou plutôt enrichi) par elle. Plus on écrit, plus on a de souvenirs. " ${ }^{49}$ Grâce à ce pricipe de simultanéité, le travail de mémoire devient un travail de deuil, et les deux démarches représentent un instrument privilégié pour dégager « la complexité de l'Histoire même et du réseau relationnel qui la constitue " pour reprendre la citation de Kablitz; une oeuvre romanesque comme Histoire peut, grâce à son " quasi-passé », mieux rendre compte de l'histoire du $\mathrm{XX}^{\circ}$ siècle que " les livres d'Histoire" dont parle l'oncle du protagoniste.

Dans le passage du Jardin des Plantes (1997), consacré à la décade de Cerisy, Simon, sans autre commentaire laisse le dernier mot à Robbe-Grillet : "Il n'en reste pas moins que C.S. nous donne constamment ses référents. [...] Donc, il faut bien croire que S. accorde aux référents une importance supérieure à celle que font les autres romanciers de cette réunion. ${ }^{50} \mathrm{Si}$ l'on envisage le travail de déplacement d'une "Histoire en train de se faire » vers un travail de mémoire sur et avec cette histoire, et le titre du roman de 1967 y renvoie explicitement, une autoréférentialité ou une réflexivité sans limites semblent impossibles ; "Le quasi-passé de la fiction devient ainsi le détecteur des possibles enfouis dans le passé effectif " comme l'a formulé Ricœur. Grâce au «style abrupt, qui suggère l'inexprimé, l'arrière-plan, la complexité, qui appelle l'interprétation »(Auerbach) et avec son travail de deuil et sa mélancolie, " ce roman peut aussi être lu comme une réflexion sur l'Histoire, avec majuscule » (Nykrog). Il représente donc un nouveau type de roman " historique " "sous la forme d'une négation de la négation " (Lukacs). Ce nouveau type de roman historique n'est pas seulement nécessaire, par suite du démenti apporté par l'invention de la photographie à

${ }^{47}$ Peter Janssens, Faire l'Histoire, Claude Simon, Presses du Septentrion 1998, p. 141.

${ }^{48}$ Mireille Calle-Gruber : "Interview avec Claude Simon », dans Claude Simon, Chemins de la mémoire, PU de Grenoble 1993, p. 14.

${ }^{49}$ Ibid., p. 23.

${ }^{50}$ Claude Simon, Le Jardin des Plantes, Minuit 1997, p. 358. 
la conception de l'histoire hégélienne (celle de la fin de l'histoire) rappelé à juste titre par Peter Janssens -, ce nouveau type est devenu nécessaire et inévitable à cause de l'histoire même du $\mathrm{XX}^{\circ}$ siècle. Ce qu'on appelle en Allemagne, en général au singulier, le Zivilisationsbruch (la rupture de civilisation) a pour conséquence une déconstruction de l'histoire que la littérature a peut-être mieux pu prendre en compte que l'Histoire comme discipline... 